\title{
NUEVAS TENDENCIAS EN EL PROCESO DE FORMACIÓN Y MENTORÍA DE PROFESORES UNIVERSITARIOS NOVELES EN SU PRIMER AÑO DE DOCENCIA
}

\section{NEW TRENDS IN THE EDUCATION AND MENTORING PROCESS OF BEGINNING UNIVERSITY PROFESSORS IN THEIR FIRST YEAR OF TEACHING}

\author{
Carmen Yot Domínguez* \\ Cristina Mayor Ruiz **
}

\begin{abstract}
Resumen: En el presente artículo se describe el programa de iniciación a la docencia de la Universidad de Sevilla a partir de la propia vivencia y se proponen líneas de mejora a cada una de sus fases, entre otras: crear y dinamizar una red social donde profesores principiantes y expertos puedan interactuar y comunicarse, incorporar el blog como herramienta útil para el desarrollo de un portfolio que ayude a la reflexión sobre la práctica pedagógica y utilizar los juegos y simulaciones como una estrategia o herramienta de aprendizaje más.
\end{abstract}

Palabras claves: Profesorado principiante. Mentoría. Reflexión. Simulación. Red social. Blog. Portfolio.

\begin{abstract}
This article describes the training program for beginning teachers at the University of Seville which considers future teachers' own experience, and proposes the improvement of each of its phases, among others: the creation and strengthening of a social network where beginning teachers, and expert teachers can interact and communicate, incorporate the blog as a useful tool for the development of a portfolio that helps them reflect on their teaching practice, as well as use games and simulations as a strategy or a tool for learning.
\end{abstract}

Keywords: Beginning teacher. Mentoring. Reflection. Simulation. Social network. Blog. Portfolio.

\footnotetext{
* Personal Docente e Investigador en Formación Didáctica y Organización Educativa. Facultad de Educación. Universidad de Sevilla. E-mail: <carmenyot@us.es>.

* Professor and Researcher in Didactics and Educational Organization. Faculty of Education. University of Sevilla. E-mail: <carmenyot@us.es>.

** Profesora Titular de la Universidad de Sevilla Didáctica y Organización Educativa. Facultad de Educación. Universidad de Sevilla. E-mail: <crismayr@us.es>.

** Professor at the University of Sevilla Didactics and Educational Organization. Faculty of Education. Univeristy of Sevilla. E-mail: <crismayr@us.es>.
} 


\section{Introducción}

Los profesores universitarios principiantes nos enfrentamos en nuestro primer año de iniciación a la docencia ante una actividad para la que antes no hemos sido formados y que nunca hemos ejercido, a saber: la enseñanza.

Es por ello que, como señalan Beca y Boerr (2009, p. 110), ante la nueva actividad profesional:

expresamos, de una u otra forma, nuestra inquietud por lograr dominar los secretos del oficio; aquéllos que permiten a los profesores experimentados ser capaces de estar atentos a las necesidades de todos sus alumnos al mismo tiempo, de flexibilizar los métodos y las actividades para que todos los estudiantes puedan participar, de mantener disciplina, de utilizar el tiempo eficientemente de manera que alcance para realizar lo planificado, de atender a lo emergente y no perder el foco en el objetivo propuesto.
Los programas de inserción profesional para profesores principiantes son una verdadera alternativa para favorecer que los primeros años como docentes no sean años frustrantes, para contrarrestar las inquietudes de lo que ha sido aterriza como puedas (MARCELO GARCÍA, 2009).

\section{Vivencia del proceso de formación y mentoría a profesores principiantes de la Universidad de Sevilla}

El programa de inserción profesional a la docencia, que se describe y lleva a examen en el presente artículo, se desarrolla en la Universidad de Sevilla desde 1995 en el seno del Instituto de Ciencias de la Educación. Según ha sido publicado (MAYOR RUIZ, 2009b; MAYOR RUIZ; SÁNCHEZ MORENO, 1999; SÁNCHEZ MORENO; MAYOR RUIZ, 2006), está conformado por: los talleres de análisis, los ciclos de mejora y las sesiones formativas.

Figura 1 - Estructura del programa de Formación para Profesores Noveles Universitarios de la Universidad de Sevilla

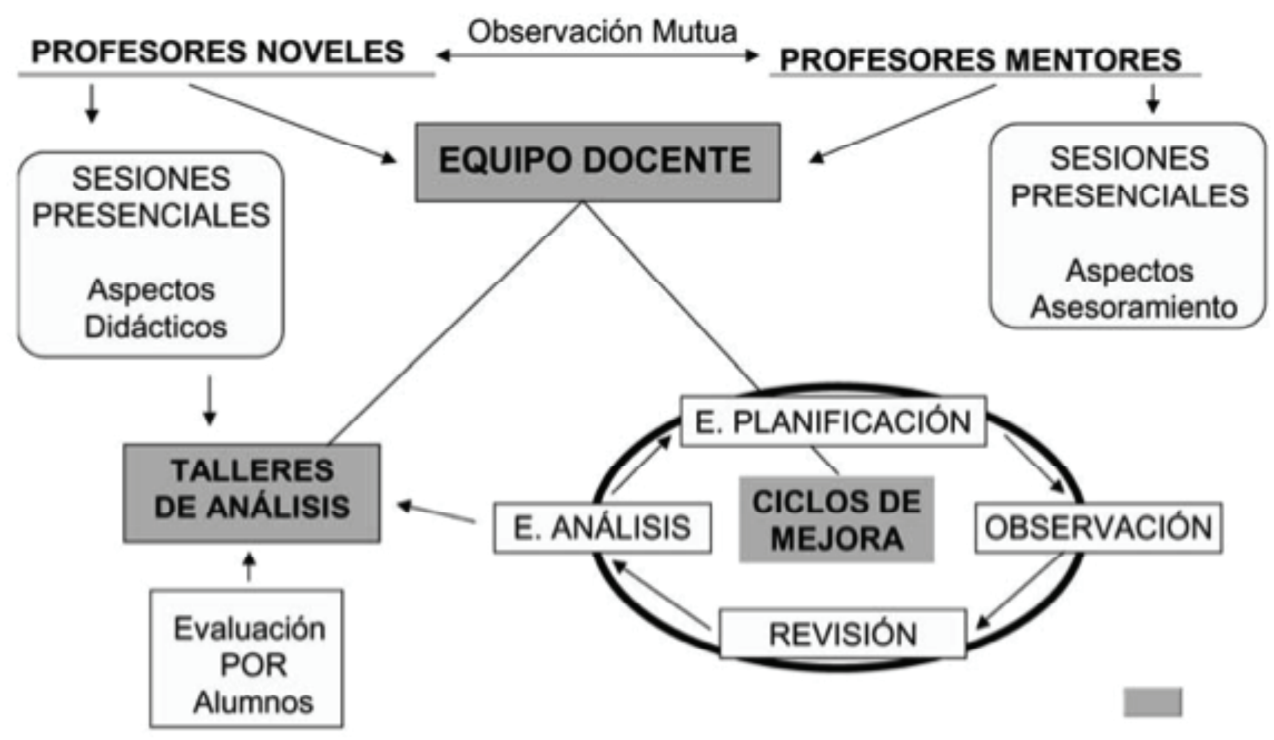


Pasamos, a continuación, a describir nuestra propia experiencia en cada una de las citadas fases.

\subsection{Participación en las sesiones formativas presenciales y online para profesores principiantes}

Bajo la asunción de que la enseñanza es una actividad profesional, lo que supone reconocer que los profesores poseen un cuerpo especializado de conocimiento (DOYLE, 1990), adquirido a través de la formación y la experiencia (CALDERHEAD, 1987), que Shulman, Wilson y Richert (1987, p. 106) definirían como conocimiento base e identificarían como imprescindible para desempeñarse con eficacia en una situación educativa o formativa dada, nos preguntamos: ¿qué tipo de conocimiento tienen los docentes para la enseñanza?

La pregunta tiene una respuesta automática que no es una aserción nueva y ha venido condicionado no sólo las prácticas formativas desarrolladas en las aulas universitarias hasta la actualidad sino también el proceso de incorporación de los profesores noveles a la docencia, a saber: la eficacia de cualquier proceso de enseñanzaaprendizaje es dependiente de lo que sabe el profesor sobre el tema que enseñará. Sin embargo, hay una diferencia enorme entre conocer un tópico o tema y saber de su enseñanza particular y las demandas para su aprendizaje (BUCAT, 2004). Como podemos entender, aunque la comprensión personal del contenido puede ser necesaria, no es una condición suficiente para enseñar. Los docentes deben encontrar vías para comunicar el conocimiento al alumnado, para ayudar a que los estudiantes lleguen a la comprensión de la materia (SHULMAN et al., 1987). Así, además de conocer los contenidos los docentes deben ser capaces de analizar un tópico hasta desmenuzarlo y hacerlo comprensible, apreciar cuál es la mejor manera de aproximarse a los contenidos, seleccionar las estrategias metodológicas adecuadas y los recursos que mayor impacto puedan tener como facilitadores del aprendizaje u organizar las ideas y tareas para los estudiantes (ZABALZA, 2004).

$\mathrm{Si}$ trasladamos las aportaciones anteriores al ámbito de la educación superior y tratamos de analizar el conocimiento base del docente universitario, lo primero que hemos de apuntar es que aunque el docente domina un ámbito específico de una ciencia carece del conocimiento pedagógico general o al menos no dispone de un amplio conocimiento pedagógico. Aún cuando deberíamos hablar de su doble identidad como experto en su disciplina y experto en la docencia de la misma, más bien hemos de afirmarque cuenta con lo que Imbernon(2000, p. 45) denominó conocimiento pedagógico vulgar, conocimiento interiorizado durante los años de alumno en las aulas y que se impone como currículum nulo dado se aprende a enseñar, más que por formación específica, de la experiencia subjetiva y siempre con la justificación de falacias del tipo "se aprende a nadar tirándose a la piscina en la primera clase" (IMBERNON, 2000; MANSO MARTÍNEZ, 1999; MAYOR RUIZ; SÁNCHEZ MORENO, 2006).

El programa de inserción profesional de la Universidad de Sevilla favoreció la formación pedagógica del profesorado novel a través de dos acciones formativas, una presencial y otra online, que nos ayudaron a vivir con relativa seguridad y autonomía nuestro primer choque con la realidad.

El programa se inició con el desarrollo de dos sesiones formativas presenciales sobre comunicación y logopedia, 
respectivamente. Ambas tuvieron lugar al inicio del curso académico y fueron impartidas por especialistas de las materias. Con posterioridad a ellas los docentes principiantes nos iniciamos en el estudio de cinco módulos de contenidos a través de la plataforma WebCT de la Universidad. Estos abordaban temáticas tan variadas como las condiciones organizativas de nuestras facultades y departamentos, los principios del aprendizaje adulto o las modalidades de tutorización posibles en el contexto universitario. Cada módulo de contenidos estaba constituido por un conjunto de documentos de lectura obligatoria $\mathrm{y}$ solicitaba el desarrollo de una tarea de aprendizaje, generalmente, de análisis de la documentación leída y de relación de las lecturas con la práctica y contexto particular de cada docente.

Según Rodríguez Espinar (2003), cualquier programa de formación debe adoptar múltiples mecanismos para su implementación y debe ofrecer una gran flexibilidad de acceso. Bien es cierto que el estudio a través de Internet, frente a la formación presencial, ha flexibilizado nuestro proceso formativo permitiéndonos el acceso en cualquier lugar y momento, favoreciendo asimismo que todos los docentes participantes en el programa pudiéramos incorporarnos a la acción formativa. En cambio, se ha podido observar que no todos hemos podido asistir a la totalidad de las sesiones presenciales, por incompatibilidad horaria en la mayoría de los casos. Es por ello que animamos a que éstas sean grabadas y puestas a disposición en la plataforma de teleformación, junto a los recursos de aprendizaje que se facilitan, para garantizar que los docentes no asistentes puedan ser partícipes también de ellas.

\subsection{El desarrollo del Ciclo de Mejora}

No es lo mismo un programa de inducción, iniciación o inserción profesional a la docencia que un programa de formación inicial o formación permanente del profesorado. No es lo mismo.

Según Smith e Ingersoll (2004), en teoría, los programas de inserción no son programas de capacitación inicial ni adicional per se sino que están diseñados para atender a los profesores, que ya han completado la formación básica y se encuentran en los inicios de la actividad profesional de enseñanza, en su conversión de estudiante a profesor.

Como reconoce Marcelo García (2007), la idea de que los profesores principiantes requieren un sistema estructurado para apoyar su entrada en la profesión deja de considerarse como algo accesorio y pasa a entenderse como un elemento central en el proceso de retención del profesorado principiante y de mejora de la calidad de su enseñanza. Ahora tenemos mayor conciencia de que el inexistente o pobre acompañamiento inicial tiene gran impacto, entre otros, en la percepción que los profesores principiantes tienen de sus propias habilidades docentes y sus relaciones con los estudiantes y en cómo se sienten y entienden su profesión (SHARP, 2006).

En los programas de inserción profesional, aún cuando pueden darse diferentes elementos, uno esencial es el proceso de asesoramiento. El apoyo y la orientación ayudan a los profesores que se inician en la enseñanza a afrontar mejor sus primeros años profesionales y a superar sus inseguridades de adaptación, acomodación y de tránsito (SÁNCHEZ MORENO et al., 2003).

Hobson, Ashby et al. (2009) definen la mentoría como el apoyo que recibe un 
principiante o un profesional con poca experiencia de un profesional experto (mentor), pensado principalmente para ayudar al desarrollo de la experiencia y facilitar su iniciación en la cultura de la profesión (en este caso, la enseñanza) y en el contexto específico local (en este caso, la universidad).

Numerosos son los autores que han analizado la relación que debe establecerse entre mentor y principiante en un proceso de asesoramiento (FEIMAN-NEMSER, PARKER; ZEICHNER, 1993; GRATCH, 1998). Feiman-Nemser (2001) afirma que los mentores deben asistir las preocupaciones y preguntas presentes de los profesores noveles sin perder de vista el objetivo a largo plazo de desarrollo del profesorado, deben interactuar con los principiantes de manera que fomenten una actitud inquisitiva y deben cultivar las habilidades y hábitos que le permitan a los docentes aprender en y desde su práctica. Ellos deben usar sus conocimientos y su experiencia para crear oportunidades y condiciones que ayuden significativamente al aprendizaje del novel. Es por ello que la mentorización debe ser protagonizada por profesores expertos y experimentados, preocupados por la mejora de la docencia y capaces de redirigir la variedad de necesidades del profesor principiante en áreas como las curriculares, psicológicas, logísticas y de gestión de la clase (MAYOR RUIZ, 2009a).

\subsubsection{La constitución del equipo docente}

El equipo docente constituido en el departamento de Didáctica y Organización Educativa de la Facultad de Ciencias de la Educación ha estado conformado por un único docente principiante y un único mentor.
La formación del equipo docente surge del interés del propio profesor novel de mantener relación de asesoramiento con el profesor experto seleccionado durante su primer año de iniciación a la docencia y de la voluntariedad de este último a ejercer de mentor. Existiría en el profesor principiante la plena seguridad de que se trataría de una relación basada en la confianza, la empatía y el respeto y que los encuentros favorecerían su aprendizaje puesto que observa en el docente experto a un profesional que goza de amplia experiencia y que manifiesta sensibilidad ante la docencia y sus problemas. Ambos, novel y experto, asimismo poseemos similares estilos de enseñanza y mostramos interés por la innovación en el proceso de enseñanza-aprendizaje.

\subsubsection{El Ciclo de Mejora y la intervención del mentor}

Asumiendo que las actividades de mentoría pueden variar, entre otros, en función de las características del equipo docente constituido, la disponibilidad tanto de los profesores principiantes como del mentor, de la relación establecida entre ambos, de los objetivos que se persiguen y las estrategias definidas para su consecución, se relacionan a continuación cuales de las principales tareas que Beca y Boerr (2009, p. 116) le asignaron al mentor fueron puestas en práctica:

1. Acompañar al novel en su proceso de inserción al contexto tomando en cuenta todos los elementos que caracterizan la institución, su cultura, las características de los alumnos, así como el contexto geográfico, social y cultural.

2. Observar la intervención del profesor principiante en clases y fomentar la reflexión y el pensamiento crítico sobre

Olhar de professor, Ponta Grossa, 15(2): 297-314, 2012. Disponível em <http://www.uepg.br/olhardeprofessor> 
su propio ejercicio para que esta práctica se convierta en una forma permanente de trabajo pedagógico que le permita aprender de los errores.

3. Colaborar en la construcción de nuevas creencias y hábitos a partir de su propio trabajo y revisar las creencias que provienen de su experiencia como estudiante.

Principalmente, el Ciclo de Mejora se vio reducido a dos extensos encuentros entre el profesor mentor y principiante en los que 1) se llevó a examen la planificación realizada por el profesor novel previo al inicio del curso y 2) se visualizaron y analizaron las sesiones de clases del profesor principiante grabadas.

Sin lugar a dudas, el segundo de los encuentros fue el más fructífero e interesante. Fue una llamada de atención y el primer momento de toma de conciencia de la relevancia de la reflexión sobre la práctica pedagógica como ejercicio no sólo de revisión de la practica sino también de aprendizaje.

Según Day (2006), los profesores críticamente reflexivos entienden y responden a las conexiones entre lo que hacen en clase, las alternativas y los contextos sociales, culturales y políticos que interactúan con sus alumnos y su trabajo, saben cómo asumen su trabajo y quiénes son ellos. El proceso de mentoría contribuyó a la formación del novel como profesional reflexivo, al hacer, en la línea de lo establecido por Gomes Lima (2002), que volviera a pensar su práctica pedagógica, se cuestionara las dimensiones de su propio conocimiento y aprendiese que la realidad en el aula es única.

Al Ciclo de Mejora cabe hacérsele dos propuestas, al margen de las que en próximos apartados se relacionan:
1. Exigir y animar a los profesores principiantes a observar a sus respectivos mentores en clase y favorecer que se abran vías de reflexión sobre lo visionado.

2. Utilizar sistemas de grabación menos disruptivos. La presencia de una persona al final de la clase grabando los movimientos de los docentes contribuye a crear tensión y nerviosismo en el novel, que presta excesiva atención a qué se está registrando y pierde naturalidad en sus actuaciones. Cheung Kong (2010) nos describe el sistema de video que desarrollaron para permitir a los propios docentes grabar su desempeño en el aula y luego recuperar los videos para la autorreflexión. El sistema consta de dos partes, un sistema de grabación de video de automonitoreo para grabar en el aula y un sistema de recuperación de vídeo online para la autorreflexión a partir de unos formularios que se debían responder antes y después del visionado en línea de los archivos de video.

\subsection{Dificultades para el desarrollo del taller de análisis}

Como describen Sánchez Moreno y Mayor Ruiz (2006, p.?), los Talleres de Análisis debían de haberse constituido en la estrategia de trabajo colaborativo entre iguales, entre noveles:

Los Talleres de Análisis los entendemos como grupos de profesores que se constituyen como unidades de trabajo colaborativo en las que el eje fundamental es el análisis y la reflexión sobre la práctica docente [...] A la hora de iniciar el trabajo en estos talleres, los profesores disponen de varias posibilidades: o bien se comienza trabajando aspectos derivados de los análisis llevados a cabo en los diferentes Ciclos de Mejora en los que 
ha participado cada uno de los profesores noveles que integran el Taller o también se puede iniciar el trabajo con la evaluación que de cada uno de los profesores hagan sus alumnos.

El intercambio de reflexiones entre docentes que comparten la casuística de encontrarse en los primeros años de iniciación a la docencia y pertenecer a un mismo departamento es una estrategia enriquecedora, mas no fue posible llevarla a la práctica dado que el equipo de trabajo constituido estaba conformado por un único profesor principiante. Sería pertinente plantearse la posibilidad de que los Talleres de Análisis fuesen conformados por áreas de conocimiento para solventar esta dificultad de desarrollo.

\section{Resultados}

Los resultados de la evaluación de la iniciativa de formación y mentoría a profesores noveles universitarios a lo largo de los años ponen de manifiesto que los docentes principiantes demandamos (SÁNCHEZ MORENO; MAYOR RUIZ, 2006): 1) apoyo emocional que nos aumente la autoestima y nos confiera seguridad en aquello que hacemos y 2) orientación y apoyo en el ámbito estrictamente profesional, tanto en las tareas de enseñanza como en las de gestión, y en las relaciones interpersonales.

Conocedores de que ambas peticiones vienen siendo cubiertas por el programa de iniciación a la docencia y con la certeza de que

los profesores principiantes aún cuando estén muy bien preparados durante la formación inicial requieren de apoyo específico en su tránsito hacia el ejercicio profesional. (BECA; BOERR, 2009, p. 118).

buscando, principalmente, encontrar herramientas útiles de ayuda para reducir la tendencia al ensayo y error en el diseño e implementación de un programa docente y controlar el estrés de la transición propio de esta etapa.

Una vez inmersos en el Ciclo de Mejora, pudimos comprobar que había dos cuestiones claves que requerían formación adicional y que al docente principiante preocupaba sobremanera: no promover la participación del alumnado cuando se dan situaciones de interrogación didáctica en clase (MORATA SEBASTIÁN; RODRIGUEZ SÁNCHEZ, 1997) y controlar el desarrollo de los debates una vez estos iniciados.

En síntesis el programa ha favorecido, sin lugar a dudas, a) la autoevaluación de las propias necesidades formativas y la evaluación por parte de los colegas de la práctica pedagógica, b) el descubrimiento de la reflexión como estrategia de aprendizaje y reconocimiento de los puntos de mejora de la enseñanza, c) el reconocimiento de la necesidad de abrir el aula para conocer qué están haciendo los docentes, y d) el hallazgo de los docentes expertos como fuente de aprendizaje y guía u orientación.

\section{Propuestas de mejora y nuevas tendencias}

A continuación se detallan nuevas tendencias en el ámbito de la formación que sin lugar a dudas pueden contribuir a mejorar el desarrollo del programa de inserción profesional a la docencia descrito. La reflexión sobre cada una de las tendencias se ha realizado sobre la base de nuestra experiencia en el citado programa. 


\subsection{El portfolio web para favorecer la práctica reflexiva en los Ciclos de Mejora}

El portfolio es una herramienta de valiosa ayuda en el desarrollo profesional docente por dos razones importantes (SELDIN, MILLER; SELDIN, 2010): 1) el nivel de inversión personal en su preparación en tiempo, energía y compromiso es alto y esto es una condición necesaria para el cambio, y 2) la preparación del portfolio mueve a muchos docentes a reflexionar sobre su enseñanza profundamente.

El portfolio docente nace, según Atienza (2009), como una forma de dar cabida a las distintas formas en que el profesor se acerca a reflexionar sobre su enseñanza con el fin de descubrir su propia identidad como docente y de transformarla, en caso de que lo estime oportuno. Y esta parece ser su cualidad distintiva, a saber: la reflexión al servicio de la toma de conciencia de la propia competencia docente y de la disposición al cambio.

El portfolio permite a los docentes (SELDIN, MILLER; SELDIN, 2010): describir las circunstancias particulares de sus materias, explicar el uso de estrategias y métodos específicos y proporcionar pruebas convincentes de que ellos son eficaces, las cuales, según Fernández (2004), oscilan desde un relato reflexivo que describa su filosofía personal sobre el aprendizaje y la enseñanza, así como sus objetivos, estrategias, metodologías, etc.; una declaración personal en la que se describa sus objetivos de mejora en la enseñanza para los próximos cinco años o la descripción de pasos dados para evaluar y mejorar lo que uno está enseñando, hasta declaraciones de colegas que han observado al profesor en el aula o de los alumnos sobre la calidad de su enseñanza.
Un portfolio no es una versión redactada de un currículum vitae, un diario docente o una recopilación de apuntes ni mucho menos un cúmulo de vanidad, una exhibición de logros, pero tampoco una ocasión para lamentarse de lo mal docente que uno puede llegar a verse (ATIENZA, 2009).

El portfolio es un vehículo para la reflexión personal y profesional con miras hacia el cambio en la práctica docente. El portfolio permite al profesorado reconocer su productividad en el ejercicio de la docencia, contextualizar su experiencia, sistematizar el trabajo y a partir de ahí buscar alternativas para cumplir con los objetivos del curso. Pero para ello debe no concebirlo como repositorio de materiales y registros audiovisuales del trabajo docente y dar el siguiente paso: elaborar un texto o ensayo reflexivo sobre el trabajo realizado (ARROYO, 2002).

Según Fernández (2004), es de utilidad por tanto para:

1. Recoger y presentar evidencias y datos concretos sobre la efectividad de su enseñanza.

2. Reflexionar sobre aquellas áreas de su enseñanza que necesitan mejorar.

3. Tener un documento con el que conocer cómo ha evolucionado su enseñanza en el tiempo.

4. Compartir sus conocimientos y su experiencia con los profesores de su departamento o centro más jóvenes.

El blog es una herramienta 2.0 que se presta al desarrollo de portfolios electrónicos dadas sus siguientes características (CHUANG, 2008): la publicación instantánea de texto y gráficos en la web sin la necesidad de conocimientos de programación 
sofisticada, los mecanismos con que las personas podrán formular observaciones o comentarios a cada entrada con facilidad y las oportunidades para archivar post pasados por fecha, para establecer hipervínculos a otros bloggers así como embeber recursos multimedias.

Deng y Yuen (2011) entienden que el uso de los blogs en el contexto de la formación docente tiene repercusión en cuatro áreas: la auto- expresión, la autorreflexión, la interacción social y el diálogo reflexivo. La auto-expresión y la autorreflexión se centran en los procesos individuales de exploración, descripción, análisis y deliberación. La auto-expresión implica dar a conocer nuestros pensamientos y emociones, así como la grabación de las experiencias propias. La autorreflexión se mueve hacia la búsqueda de significado de estas experiencias. Por otra parte, la interacción social y el diálogo reflexivo constituyen la dimensión comunitaria que tiene que ver con los procesos sociales de intercambio de ideas y apoyo. Estas cuatro áreas fueron estructuradas atendiendo a los tres grandes tipos de comportamientos en los blogs: escribir, leer y comentar.
En términos más concretos, escribir en un blog permite expresar sentimientos y pensamientos personales y fomenta la autorreflexión. Desde la perspectiva de los lectores del blog, las historias reales relatadas en los blogs pueden servir de estímulo para la reflexión o como fuente de inspiración. En la dimensión comunitaria, a través de interacciones con los compañeros, proporciona un lugar alternativo para el intercambio de apoyo social.

En resumen, promover que cada docente principiante cree su blog docente y comience la elaboración de su propio portfolio ayudará al desarrollo del proceso de reflexión dado en el Ciclo de Mejora. Al mismo tiempo animar a la lectura de los blog de otros compañeros/as del programa novel e incidir en que intercambien comentarios contribuye a la consecución de los objetivos de los Talleres de Análisis.

Figura 2 - Representación de Deng y Yuen (2011)

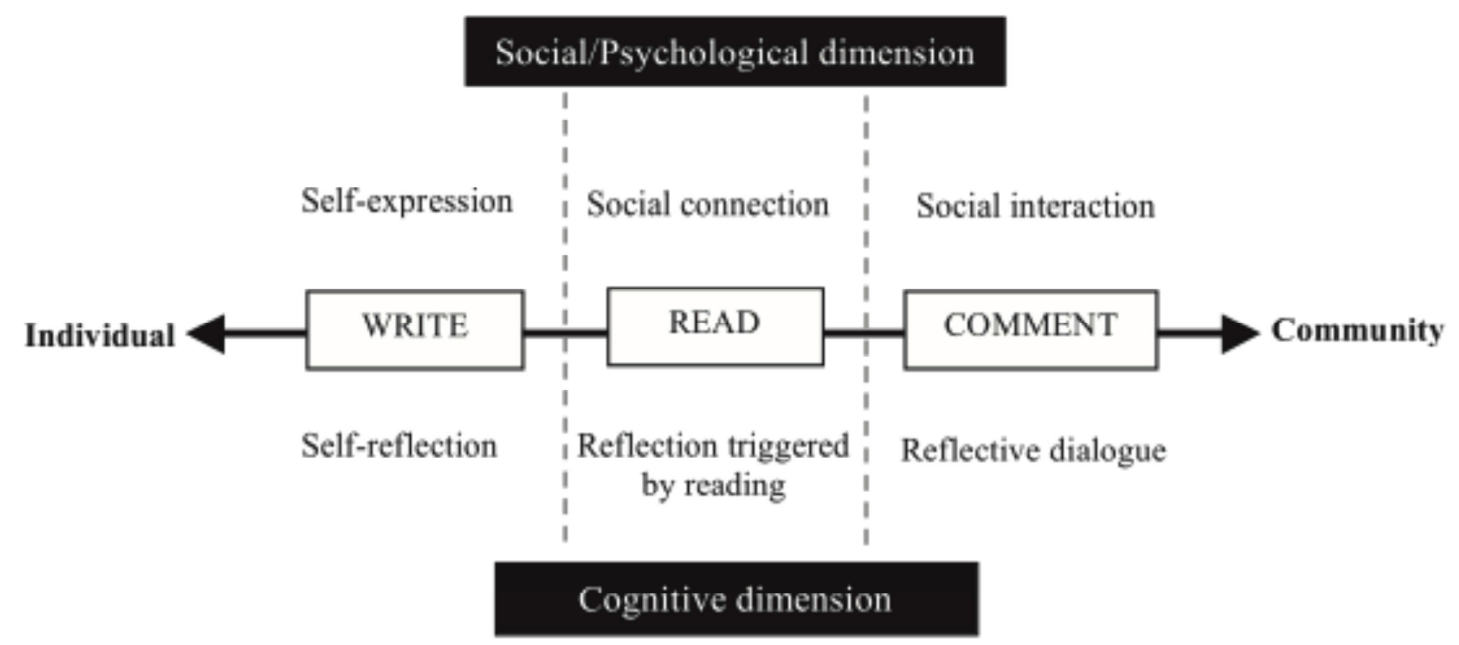




\subsection{La red social para suplir las dificultades de desarrollo del Taller de Análisis}

Los Talleres de Análisis están pensados para favorecer la construcción colaborativa de la profesionalidad docente. Mas si los equipos docentes en el seno de cada departamento están constituidos por un único profesor novel, su puesta en práctica de partida se ve dificultada.

En la actualidad en Internet podemos encontrar diferentes redes sociales docentes como esta que ilustramos y que la conforman docentes y expertos interesados por la iniciación a la docencia.

Figura 3 - Red social sobre Iniciación a la docencia

\section{Red sobre Iniciacion a la docencia}

Profesorado principiante e insercion profesional

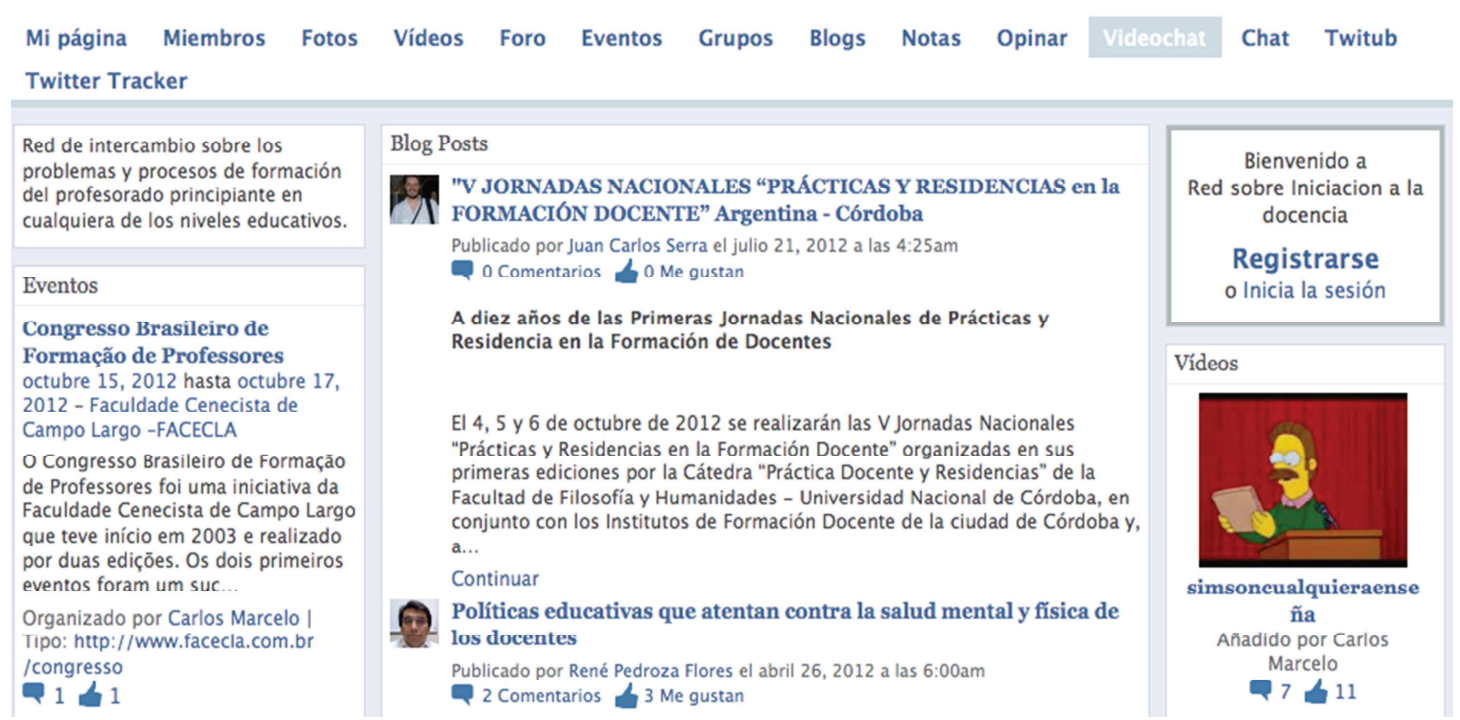

DeWert et al. (2003) describen el diseño, implementación y resultados de una comunidad online para profesores principiantes compuesta por profesores noveles, profesores expertos y miembros de la Facultad de Educación cuya finalidad era establecer un vínculo más fuerte entre los años de pregrado y de inducción. Los autores observaron que la comunidad online alteró la forma en que los nuevos docentes se comunican entre sí (apoyo mutuo), así como con otros profesionales (consulta) dado entre otras ventajas la supresión de barreras de tiempo y distancia y la comodidad y flexibilidad de comunicarse en cualquier momento y desde cualquier lugar.

Mochizuki y Kitazawa (2009), quienes crearon una comunidad de aprendizaje usando el servicio de las redes sociales para que los profesores noveles pudieran comunicarse en línea y recibir apoyo social, afirman que el intercambio de experiencias y opiniones promovió que los profesores reflexionaran sobre su experiencia de trabajo diario. Además, el conocimiento práctico de los compañeros/as sirvió como información útil para la formación de los miembros. 
Las principales potencialidades de la existencia de una red social de docentes en Internet, de hecho, según Area (2011), son las siguientes:

- Permite “hacer público" al grupo o colectivo de docentes en el ciberespacio;

- Permite que la comunicación entre los miembros de la red pueda realizarse en cualquier momento y desde cualquier lugar;

- Permite compartir e intercambiar materiales y recursos elaborados individualmente;

- Permite generar documentos y/o materiales mediante un proceso de trabajo colaborativo en un periodo de tiempo dado;

- Permite que cada docente pueda reconstruir personalmente su experiencia y comunicarla a los demás a través de blogs o diarios digitales;

- Permite la creación de tablones/canales de noticias de interés conjunto para el grupo.

\subsection{Los juegos y simulaciones como estrategia formativa a considerar}

En opinión de Prensky (2001), la revolución formativa y del aprendizaje no es, a pesar de lo que muchos predicen, la formación a través Internet, aún cuando es importante. La verdadera revolución del aprendizaje del siglo XXI es que el enorme muro que ha separado el aprendizaje y la diversión, el trabajo y el juego, en los últimos cientos de años, está finalmente comenzando a temblar y se viene abajo para beneficio de todos. Y la buena noticia es que cuando lo haga vamos a encontrar que adicionar la diversión al proceso de enseñanzaaprendizaje no sólo hará que el aprendizaje y la formación sean mucho más agradables y atractivos, sino mucho más eficaces también.

Tanto las simulaciones como los juegos pueden emplearse como herramientas de formación y educación. Las principales ventajas percibidas de la utilización de los juegos y las simulaciones son (FREITAS, 2006): 1) aumento de los niveles de motivación de los alumnos, 2) aumento de las tasas de retención del alumnado a través del disfrute, 3) potencial para ampliar la participación y el uso del aprendizaje colaborativo y 4) la eficacia del aprendizaje mediante la experiencia.

Los juegos conectan los específicos contenidos y habilidades con un ambiente agradable donde el alumno es capaz de tocar, probar, equivocarse y aprender. Los juegos fortalecen los procesos cognitivos y mejoran ciertas habilidades y capacidades, ofrecen retroalimentación en tiempo de ejecución y permiten que el flujo de aprendizaje se adapte sobre la marcha. También proporcionan un buen vehículo para establecer relaciones sociales (BURGOS, TATTERSALL; KOPER, 2007).

En cuanto a la simulación, citamos algunas de sus ventajas específicas (MAHBOUBIAN, 2009): a) cuanto más se asemeje la simulación al ambiente real de aprendizaje mayor será la retención, b) las simulaciones proporcionan un ambiente seguro en el que cometer errores, c) las simulaciones permiten aprender sin necesidad de equipos costosos, d) la creación de la simulación puede ayudar a racionalizar los procesos que se enseña (es decir, mejoras en el proceso se hacen a menudo cuando se crean simulaciones); e) las simulaciones bien diseñadas a menudo reducen el tiempo de aprendizaje de manera significativa y f) las simulaciones permiten la práctica de procedimientos peligrosos. 
ClassSIMs es un ejemplo de simulación empleada para formar a los docentes en el periodo de prácticum. Los primeros resultados de su utilización nos sugieren que:

1. Los profesores encuentran que ClassSIM es un entorno seguro en el que ellos son capaces de obtener una visión inicial de la profesión, un mayor entendimiento del rol del maestro, y hacer importantes conexiones entre la teoría y la práctica (CARRINGTON, KERVIN; FERRY, 2008);

2. Los docentes usuarios llegan a comprender las situaciones complejas que tienen lugar en el aula. Han podido entrenarse en ellas dándoles la oportunidad de frenar o acelerar los acontecimientos del aula, revisar y reflexionar sobre los puntos de decisión críticos y reproducir eventos a la luz de nuevos conocimientos. (FERRY et al., 2004).

Según Ferry et al. (2004), la citada simulación permite al usuario tomar el papel del profesor de un aula de Educación Infantil. El usuario se encuentra, así, con una clase de infantil (5-6 años) que consta de 26 estudiantes en la que está obligado a tomar una serie de decisiones sobre la gestión del aula, los estudiantes y los eventos aleatorios que ocurren típicamente durante una lección. En otras ocasiones, están obligados a tomar decisiones acerca de la secuencia de enseñanza. Cada una de estas decisiones tiene el potencial de influir en las decisiones posteriores en cada una de estas áreas descritas.

Al margen de los episodios, la simulación incorpora una herramienta que permite al usuario reflexionar en la acción, reflexionar sobre la acción y reflexionar sobre la reflexión en la acción, a saber: Espacio de Pensamiento. Ésta está siempre disponible, todo el tiempo de duración de la simulación, favoreciendo que el usuario pueda reflexionar sobre los problemas dentro del aula, articular sus fundamentos en los puntos de decisión y de identificar las influencias subyacentes que afectan el uso del entorno virtual de aprendizaje, así como registrar de su aprendizaje profesional (CARRINGTON, FERRY; KERVIN, 2006).

Otro ejemplo de simulador es Classroom SIM: Discipline Strategies 6-8, distribuido por aha! Process, Inc. como complemento al libro Working with Students: Discipline Strategies for the Classroom del Dr. Ruby K. Payne. 
Figura 4 - Classroom SIM

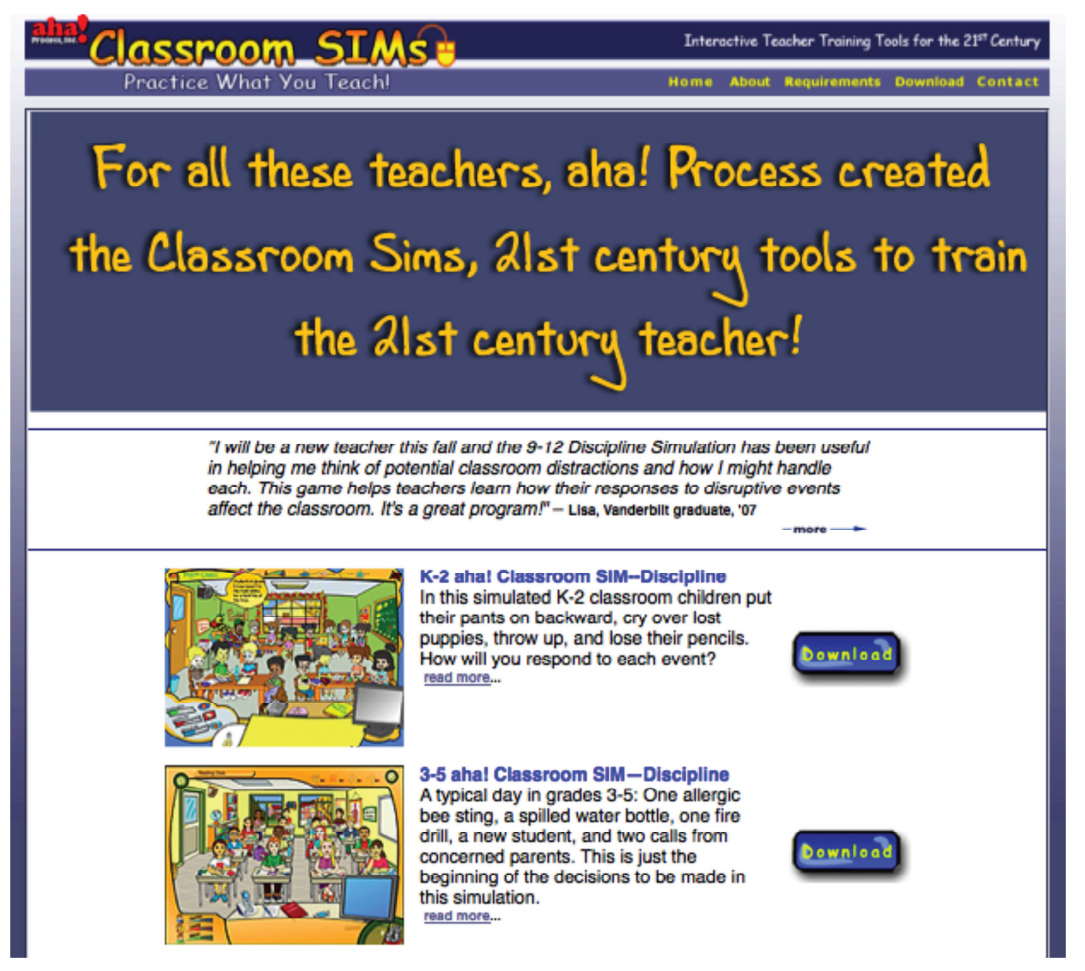

En el marco del Programa Internet en el Aula, la entidad pública Red.es también En este caso se aborda la resolución de conflictos en el contexto del aula. ha desarrollado un simulador para docentes.

Figura 5 - Simulador del aula

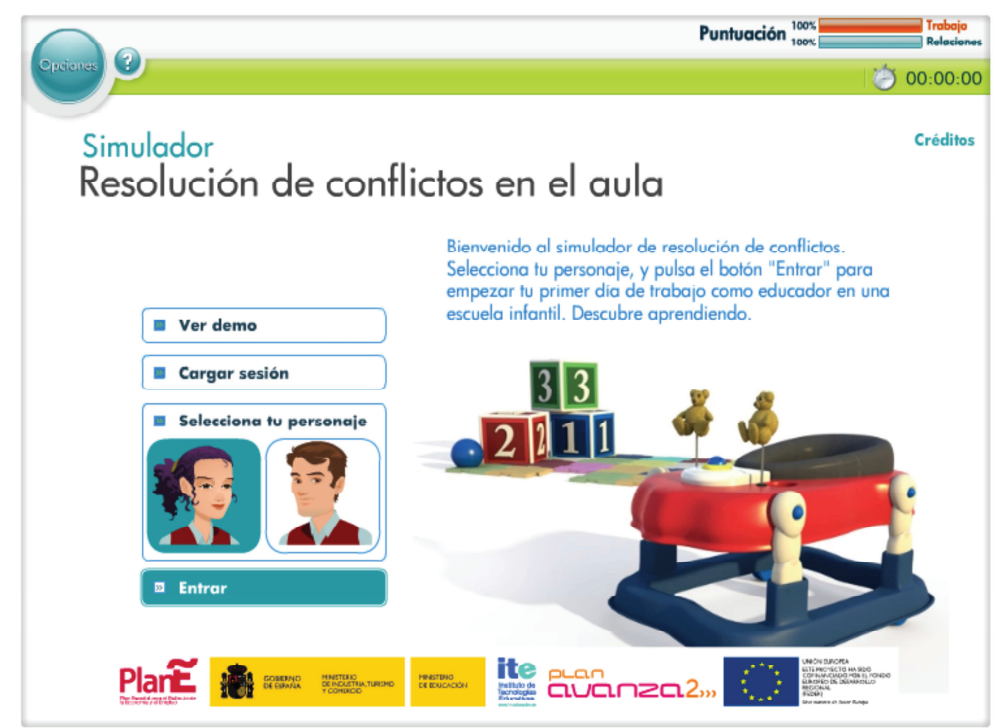


Por otra parte, Cheong (2010) investiga el efecto que ejerce la práctica docente en Second Life puede tener en el cambio de eficacia en la enseñanza de los futuros docentes.

Second Life es de los mundos virtuales el que mayor atención ha recibido. Los mundos virtuales 3D representan la tecnología emergente, mas ha habido tecnologías que la han precedido, como los simuladores 3D (CRELLIN, DUKEWILLIAMS; CHANDLER; COLLINSON, 2009), los Dominio de Multiusuario (MUD) y los MUD Orientado a Objetos (MOO) (Dickey, 1999). Según Dieterle y Clarke (2009), fueron los avances en el poder computacional y la conectividad de red los que impulsaron la evolución de los MUDs, dando lugar a diversas interfaces de ordenador como los MOOs (MUD orientado a objetos), los MMORPGs (Juegos Online de Rol Multijugador Masivos) y los MUVEs (Entornos Virtuales Multiusuario), entre otros.

Definidos los mundos virtuales (MUVEs) como red síncrona y persistente de personas, representadas como avatares, facilitada por la conexión de los ordenadores (BELL, 2008), éstos pueden aparecer bajo diversas formas $\mathrm{y}$, sin embargo, poseer un número de características recurrentes (WARBURTON, 2009): persistencia del ambiente, facilidad para que múltiples usuarios participen simultáneamente, encarnación virtual bajo la forma de avatar (pero sin asumir roles ficticios o predeterminados ni tener el propósito de cubrir tareas a él asignadas como en los MMOGs), interacciones que ocurren entre los usuarios y los objetos en un ambiente tridimensional, inmediatez de la acción tal que las interacciones ocurren en tiempo real y semejanzas al mundo real como topografía, movimiento y física que proporcionan la ilusión de estar allí.
Parece claro que los mundos virtuales serán herramientas de interacción social y conocimiento ampliamente difundidas y que se convertirán en otra parte del sistema socio-técnico que la gente utilizará para la enseñanza y el aprendizaje en el futuro previsible (JARMON; LIM; CARPENTER, 2009). Dalgarno y Lee (2010) exploran las características de los entornos de aprendizaje virtuales 3D. Los beneficios de aprendizaje quedan resumidos en los que sigue:

- Los entornos virtuales de aprendizaje 3D pueden ser utilizados para facilitar tareas de aprendizaje que conducen al desarrollo de una mayor representación del conocimiento espacial del dominio explorado;

- Los entornos de aprendizaje virtuales 3D pueden ser utilizados para facilitar tareas de aprendizaje experienciales que son impracticables o imposibles de realizar en el mundo real;

- Los entornos virtuales 3D pueden ser utilizados para facilitar tareas de aprendizaje que conducen a una mayor motivación intrínseca y al compromiso;

- Los entornos 3D pueden ser utilizados para facilitar tareas de aprendizaje que conducen a mejorar la transferencia de conocimientos y habilidades a situaciones reales a través de la contextualización del aprendizaje;

- Los entornos virtuales 3D pueden ser utilizados para facilitar tareas que llevan a un más rico y/o eficaz aprendizaje colaborativo que el que es posible con los entornos 2D.

Al margen de lo citado hemos de reconocer, dada nuestra experiencia, que ciertamente hay una gran cantidad de posibilidades educativas y de formación a ocurrir en entornos virtuales multiusuarios 
(MUVEs), sobre todo, como señala Berge (2008), cuando los objetivos implican role playing, simulación e interacción entre pares.

\section{En síntesis}

Entendemos que el programa puede ser enriquecido si se atiende a las siguientes líneas de mejora:

1. Crear y dinamizar una red social donde profesores principiantes y expertos puedan interactuar y comunicarse de forma continuada y con total flexibilidad geográfica. Ésta puede constituirse en herramienta a) de rápido asesoramiento de un experto o entre iguales $\mathrm{y} b$ ) de trabajo colaborativo entre los docentes principiantes, y si así se establece en grupos de una misma Facultad o área de conocimiento;

2. Incorporar el blog como herramienta útil para el desarrollo de un portfolio que ayude a la autorreflexión sobre la práctica pedagógica. Asimismo al hacer visible la realidad del aula a otros docentes los cuales pueden interesarse por lo que hacemos y aportarnos sus apreciaciones y valoraciones, ayudará a tomar conciencia de lo que se puede mejorar y favorecerá el aprendizaje a partir de los otros;

3. Utilizar los juegos y simulaciones como una estrategia o herramienta de aprendizaje más. Si es posible aprender jugando en otros niveles educativos, también lo es en la formación del profesorado.

\section{Agradecimientos}

Dedicar estas últimas palabras a quien fue mentor, D. Carlos Marcelo García. A él se agradece su apoyo y siempre disposición.

\section{Referencias}

AREA, M. Las redes sociales en internet como espacios para la formación del profesorado. Razón y palabra, n. 63, 2011.

ARROYO, G. C. Consideraciones sobre el uso del portafolio de desempeño docente en educación superior. Acción pedagógica, $v$. 11, n. 2, 2002.

ATIENZA, E. El portafolio del profesor como instrumento de autoformación. Revista de didáctica ELE, n. 9, 2009.

BECA, C. E.; BOERR, I. El proceso de inserción a la docencia. In: MEDRANO, C., V.; VAILLANT, D. (Eds.). Aprendizaje y desarrollo profesional docente Madrid: OEI, 2009, p. 109-118.

BELL, M. W. Toward a definition of "virtual worlds". Journal of Virtual Worlds Research, v. 1, n.1, 2008. http://journals.tdl. org/jvwr/article/viewFile/283/237

BERGE, Z. L. Multi-user virtual environments for education and training?: a critical review of second life. Educational technology: the magazine for managers of change in education, v. 48, n. 3, p. 27-31, 2008.

BUCAT, R. Pedagogical content knowledge as a way forward: applied research in chemistry education. Chemistry education: research and practice, v. 5 , n. 3 , p. 215228, 2004.

BURGOS, D.; TATTERSALL, C.; KOPER, $\mathrm{R}$. Re-purposing existing generic games and simulations for e-learning. Computers in Human Behavior,v. 23, n. 6, 2007.

CALDERHEAD, J. Exploring teacher's thinking. London: Cassell, 1987.

CARRINGTON, L.; FERRY, B.; KERVIN, L. K. Cognitive tools of classSim: building 
connections between theory and practice. Paper presented at the Australian Association for Research in Education Conference 36th Annual Conference, Adelaide, South Australia, 2006.

CARRINGTON,L.; KERVIN,L.K.; FERRY, $B$. Virtual practicum experiences to build professional identity. Paper presented at the 16th International Conference on Computers in Education, Taiwan, 2008.

CRELLIN, J.; DUKE-WILLIAMS, E.; CHANDLER, J.; COLLINSON, T. Virtual worlds in computing education. Computer Science Education, v. 19, n. 4, p. 315-334, 2009.

CHEONG, D. The effects of practice teaching sessions in second life on the change in pre-service teachers' teaching eficacy. Computers \& Education. v. 55, p. 868-880, 2010.

CHUANG, H. Perspectives and issues of the creation for weblog-based electronic portfolios in teacher education. British Journal of Educational Technology, v. 39, n. 1, p. 170-174, 2008.

DALGARNO, B.; LEE, M. J. W. What are the learning affordances of 3-D virtual environments? British Journal of Educational Technology, v. 41, n. 1, p. 1032, 2010.

DAY, C. Pasión por enseñar: la identidad personal y profesional del docente y sus valores. Madrid: Narcea, 2006.

DENG, L.; YUEN, A. H. K. Towards a framework for educational affordances of blogs. Computers \& Education. v. 56, p. 441-451, 2011.

DEWERT, M. H.; BABINSKI, L. M.; JONES, B. D. Safe passages: providing online support to beginning teachers. Journal of Teacher Education. n. 54, 2003.
DICKEY, M. 3d virtual worlds and learning: an analysis of the impact of design affordances and limitations of active worlds, blaxxum interactive, and onlive!: traveler; and a study of the implementation of active worlds for formal and informal education. Columbus, Ohio: Ohio State University, 1999.

DIETERLE, E.; CLARKE, J. MultiUser virtual environments for teaching and learning. In: PAGANI, M. (Ed.), Encyclopedia of multimedia technology and networking. Hershey, PA: Idea Group, Inc, 2009.

DOYLE, W. Themes in teacher education research. In: HOUSTON, W. R. (Ed.) Handbook of research on teacher education. New York: Macmillan, 1990, p. 3-24.

FEIMAN-NEMSER, S.; PARKER, M. B.; ZEICHNER, K. Are mentor teachers teacher educators? In: MCINTYRE, D.; HAGGER, H.; WILLKIN, M. (Eds.) Mentoring: perspectives on school-based teacher education. London: Kogan Page, 1993, p. 147-165.

FEIMAN-NEMSER, S. Helping novices learn to teach: lessons from an exemplary support teacher. Journal of Teacher Education. n. 52, 2001.

FERNÁNDEZ, M. A. El portafolio docente como estrategia formativa y de desarrollo profesional. Educar, n. 33, p. 127-142, 2004.

FERRY, B. et al.. Incorporating real experience into the development of a classroom-based simulation. Journal of Learning Design, v. 1, n. 1, p. 22-32, 2004.

FREITAS, S. I. de. Using games and simulations for supporting learning. Learning, media and technology, v. 31, n. 4, p. 343-358, 2006. 
GOMES LIMA, P. El educador reflexivo: notas para la orientación de sus prácticas docentes. Educar, n. 33, p. 57-67, 2002.

GRATCH, Amy. Beginning teacher and mentor relationships. Journal of Teacher Education, 49, 1998.

HOBSON, A. J., ASHBY, P., MALDEREZ, A.; TOMLINSON, P, D. Mentoring beginning teachers: what we know and what we don't. Teaching and Teacher Education, n. 25, p. 207-216, 2009.

IMBERNON, F. Un nuevo profesorado para una nueva universidad: ¿conciencia o presión? Revista Interuniversitaria de Formación del Profesorado, n. 38, p. 3746, 2000.

JARMON, L.; LIM, K. Y. T.; CARPENTER, B. S. Introduction: pedagogy, education and innovation in virtual worlds. Journal of Virtual Worlds Research, v. 2, n.1, 2009.

KONG, S. C. Using a web-enabled video system to support student-teachers' self reflection in teaching practice. Computers \& Education. v. 55, p. 1772-1782, 2010.

MAHBOUBIAN, M. e-Learning through business simulation softwares. Artículo presentado en The Sixth International Conference on eLearning for KnowledgeBased Society, Tilandia, 2009.

MANSO MARTÍNEZ, J. M. Profesionalización pedagógica del profesorado universitario. Revista Interuniversitaria de Formación del Profesorado, n. 34, p. 319-328, 1999.

MARCELO GARCÍA, C. Empezar con buen pie:inserción a la enseñanza para profesores principiantes. Docencia, n.33, 2007.

Los comienzos en la docencia: un profesorado con buenos principios. Profesorado, v. 13, n.- 1-, 2009.
MAYOR RUIZ, C.;SÁNCHEZ MORENO, M. Los jóvenes profesores universitarios y su formación pedagógica: claves y controversias. Revista de Educación, n. 339, p. 923-946, 2006.

El desafío de los profesores principiantes universitarios ante su formación. In: MARCELO GARCÍA, C. (Ed.). El profesorado principiante: inserción a la docencia. Barcelona: Octaedro, p. 177-210, 2009a.

El desafío de los profesores principiantes universitarios ante su formacs ante su formación. In: MARCELO GARCÍA, C. (Ed.). El profesorado principiante: inserción a la docencia . Barcelona: Octaedro, p. 177-210, 2009b.

.; SÁNCHEZ MORENO, M. Los equipos docentes: una contribución formativa a la calidad del profesorado universitario. XXI Revista de Educación, n. 1, p. 157-176, 1999.

MOCHIZUKI, T.; KITAZAWA, T. Designing a learning community for the pre-service teacher training using the social networking service. Artículo presentado en 17th International Conference on Computers in Education, Hong Kong, 2009.

MORATA SEBASTIÁN, R.; RODRIGUEZ SÁNCHEZ, M. La interrogación como recurso didáctico. Análisis del uso de la pregunta didáctica practicado en dos áreas de conocimiento en el nivel de formación profesional. Didáctica, n.9, p. 153-170, 1997.

PRENSKY, M. From digital game-based learning. U.S.A: McGraw-Hill, 2001.

RODRÍGUEZ ESPINAR, S. Nuevos retos y enfoques en la formación del profesorado universitario. Revista de Educación, n. 331, p. 67-99, 2003. 
SÁNCHEZ MORENO, M.; MAYOR RUIZ, C. Los jóvenes profesores universitarios y su formación pedagógica: claves $\mathrm{y}$ controversias. Revista de Educación, n. 339, p. 923-946, 2006.

SÁNCHEZ MORENO, M.; MURILLO ESTEPA, P.; MAYOR RUIZ, C.; RODRÍGUEZ LÓPEZ, J. Mª ALTOPIEDI, M.; TORRES GORDILLO, J. J.. El asesoramiento pedagógico para la formación docente del profesorado universitario: un reto para nuestras universidades. Artículo presentado en el $3^{\mathrm{a}}$ Congreso Nacional y $1^{\circ}$ internacional de investigación educativa "Laberintos y encrucijadas", 2003. Disponible en: <http://prometeo.us.es/idea/publicaciones/ cris/15.pdf $>$.

SELDIN, P., MILLER, J. E.; SELDIN, C. A. The teaching portfolio: a practical guide to improved perfomance and promotion/tenure decisions. San Francisco: Jossey-Bass, 2010.

SHARP, H. Examining the consequences of inadequate induction for beginning teachers. Artículo presentado en Annual Conference of the Australian Association for Research in Education, Adelaide, 2006.

SHULMAN, L.; WILSON, S.;RICHERT, A. 150 different ways of knowing: representations of knowledge in teaching. In: CALDERHEAD, J. (Ed.). Exploring teacher`s thinking London: Cassell, p. 104-124, 1987.

SMITH, T. M.; INGERSOLL, R. M. What are the effects of induction and mentoring on beginning: teacher turnover? American Educational Research Journal, v. 41, n.3, p. 681-714, 2004.

WARBURTON, S. Second life in higher education: assessing the potential for and the barriers to deploying virtual worlds in learning and teaching. British Journal of Educational Technology, v. 40, n.3, p. 414426, 2009.
ZABALZA, M. A. La enseñanza universitaria: el escenario y sus protagonistas. Madrid: Narcea, 2004.

Enviado em: 27/09/2012

Aceito em: 13/12/2012 